\title{
Teaching EEG 4: An unexpected source of slow wave activity
}

Sarma G.R.K. ${ }^{1}$, Norman $\mathrm{So}^{2}$, Soheyl Noachtar ${ }^{3}$, Elizabeth V.T. ${ }^{4}$

${ }^{1}$ Professor of Neurology, St. John's Medical College Hospital, Bengaluru, India

${ }^{2}$ Clinical Professor, Department of Neurology, University of Washington Medical Center, USA

${ }^{3}$ Professor \& Head, Epilepsy Center, Dept. of Neurology, University of Munich, Germany

${ }^{4}$ EEG Technologist, St. John's Medical College Hospital, Bengaluru, India

Case summary:

An 18 year old male presented with clonic seizures involving left side of face, tongue and upper limb of 5 years duration. The frequency has increased over years to become almost continuous, especially precipitated by action of the involved regions, like protruding the tongue or lifting the left upper limb. His EEG is shown in Figure 1.

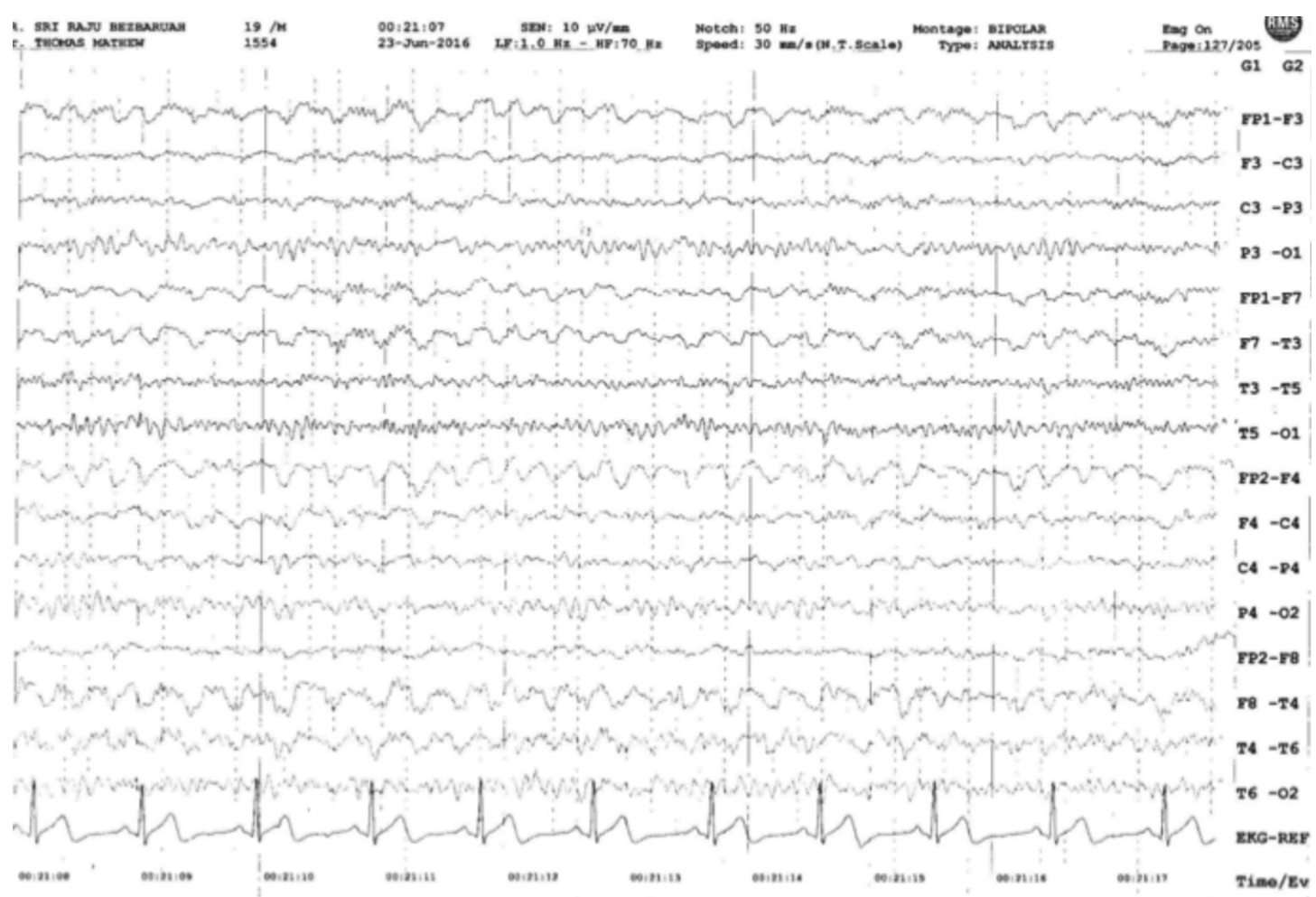

Figure 1

Questions:

1. What is the abnormality in the EEG trace shown?

2. Which artifact in this patient could produce similar activity?

3. How do you differentiate such an artifact from a cerebral slow wave activity? 
Answers:

1. The EEG shows $4-4.5 \mathrm{~Hz}$ slow wave activity predominantly involving bifrontal and temporal regions.

2. In this patient with clonic seizures of the tongue, a glossokinetic artifact must be considered. It is caused by the bioelectric dipole of the tongue due to the negative potential at the tongue tip relative to its base. It is diffusely seen with frontotemporal predominance and can closely mimic FIRDA (Frontal intermittent rhythmic delta activity).

3. Glossokinetic artifacts produced by chewing and talking are characterized by slow wave activity with prominent, superimposed muscle artifacts. However, if the muscle artifacts are absent, as in this patient, placement of infraorbital electrodes helps in identifying the source of the slow wave activity. Glossokinetic artifact would be of greater amplitude in infraorbital leads than in frontopolar leads. True cerebral slow wave activity would be of greater amplitude in frontopolar leads than in the infraorbital leads.

In this patient, the EEG in figure 1 was recorded with the protruded tongue having clonic seizures. When the tongue was retracted, the clonic seizures stopped and the EEG slow wave activity disappeared (figure 2). This confirmed that the source of bifrontal slow wave activity was a glossokinetic artifact, obviating the need for infraorbital lead placement.

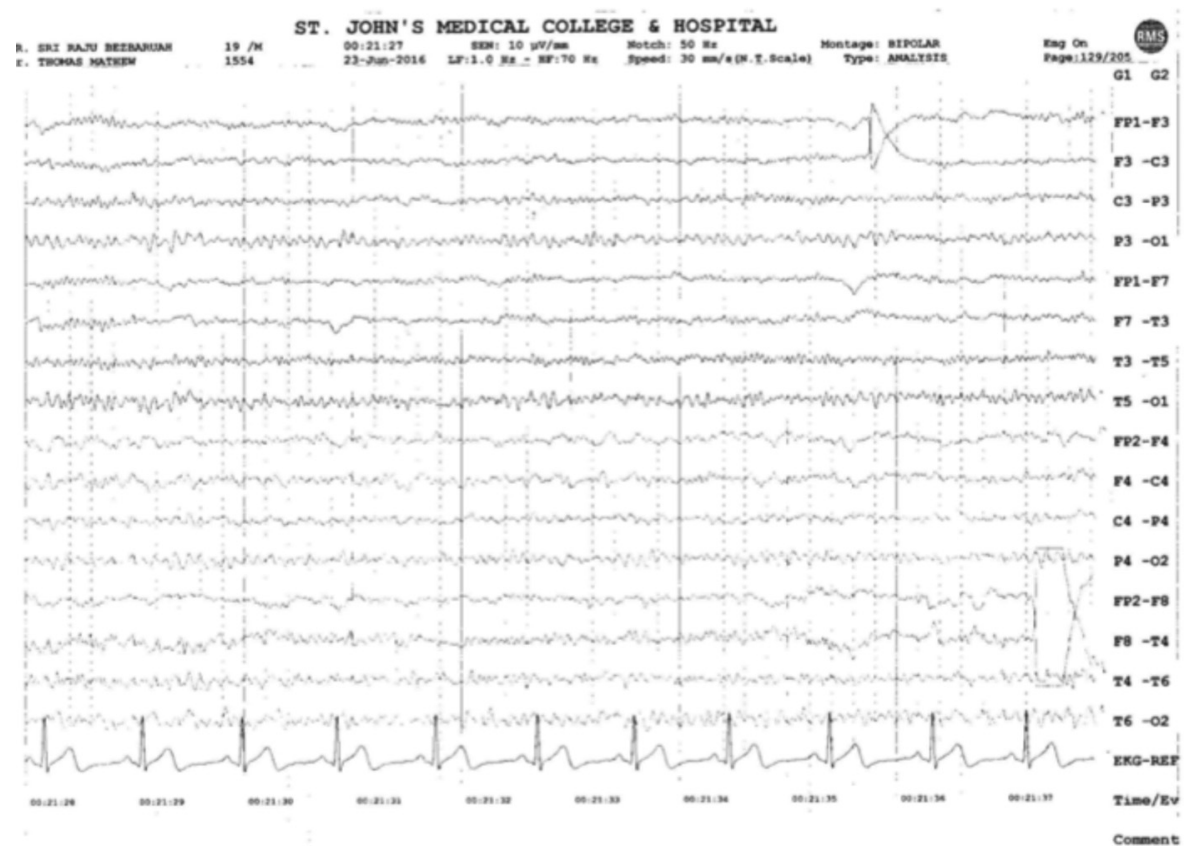

Figure 2 .

\section{REFERENCES:}

1. Dworetzky B, Herman S, Tatum WO. Artifacts of recording. In: Schomer DL, Lopes da Silva F, eds. Niedermeyer's Electroencephalography, $6^{\text {th }}$ edition. Wolters Kluwer/Lippincott Williams \& Wilkins, 2011, Chapter 13: 244-248

2. Klem GH. Artifacts. In: Ebersole JS, Pedley TA eds. Current Practice of Electroencephaography. Philadelphia, PA: Lippincott Williams \& Wilkins; 2003: 271-287

3. H.O. Luders, S. Noachtar. Atlas and classification of electroencephalography. W.B. Saunders Company, 1999; page 20. 